B. Brandolini · C. Trombetti

\title{
Comparison results for Hessian equations via symmetrization
}

Received November 25, 2005

\section{Introduction}

For a twice differentiable function $u$ defined in a domain $\Omega \subset \mathbb{R}^{n}$ the $k$-Hessian operator $(k=1, \ldots, n)$ is defined by

$$
S_{k}\left(D^{2} u\right)=S_{k}\left(\lambda\left(D^{2} u\right)\right)=\sum_{1 \leq i_{1}<\cdots<i_{k} \leq n} \lambda_{i_{1}} \cdots \lambda_{i_{k}},
$$

where the $\lambda$ 's are the eigenvalues of the Hessian matrix $D^{2} u$ of $u$ and $S_{k}$ is the $k^{\text {th }}$ elementary symmetric function. For example, for $k=1, S_{1}\left(D^{2} u\right)=\Delta u$, while, for $k=n$, $S_{n}\left(D^{2} u\right)=\operatorname{det} D^{2} u$. Equations involving these operators, and some more general equations of the form

$$
F\left(\lambda_{1}, \ldots, \lambda_{n}\right)=f \quad \text { in } \Omega,
$$

have been widely studied by many authors, who restrict their considerations to convenient cones of solutions with respect to which the operator in $(1.2)$ is elliptic. Following [25] we define the cone $\Gamma_{k}$ of ellipticity for $(1.1)$ to be the connected component containing the positive cone $\Gamma^{+}=\left\{\lambda \in \mathbb{R}^{n}: \lambda_{i}>0 \forall i=1, \ldots, n\right\}$ of the set where $S_{k}$ is positive. Thus $\Gamma_{k}$ is an open, convex, symmetric cone with vertex at the origin and

$$
\frac{\partial S_{k}}{\partial \lambda_{i}}(\lambda)>0 \quad \text { on } \Gamma_{k}, \forall i=1, \ldots, n .
$$

This implies that 1.1 is elliptic on functions $u \in C^{2}(\Omega)$ such that at each point of $\Omega$ the vector of eigenvalues of $D^{2} u$ belongs to $\Gamma_{k}$.

Following [7] we call such functions admissible (with respect to $S_{k}, k \geq 2$ ) or $k$ convex. We will denote by $\Phi_{0}^{k}(\Omega)$ the set of admissible functions that vanish on the boundary of $\Omega$.

In [12] Korevaar has shown that $\Gamma_{k}$ can also be characterized as

$$
\begin{aligned}
\Gamma_{k}=\left\{\lambda \in \mathbb{R}^{n}: S_{k}(\lambda)>0, \frac{\partial S_{k}}{\partial \lambda_{i_{1}}}(\lambda)>0, \ldots, \frac{\partial^{k-1} S_{k}}{\partial \lambda_{i_{1}} \ldots \partial \lambda_{i_{k-1}}}(\lambda)>0,\right. \\
\left.\forall 1 \leq i_{1}<\cdots<i_{k-1} \leq n\right\} ;
\end{aligned}
$$

B. Brandolini, C. Trombetti: Dipartimento di Matematica e Applicazioni "R. Caccioppoli", Università degli Studi di Napoli "Federico II", Complesso Monte S. Angelo, via Cintia, 80126 Napoli, Italy; e-mail: brandolini@unina.it, cristina@unina.it 
while in [11] Ivochkina proved that

$$
\Gamma_{k}=\left\{\lambda \in \mathbb{R}^{n}: S_{1}(\lambda)>0, \ldots, S_{k}(\lambda)>0\right\} .
$$

In this paper we deal with the following Dirichlet problem:

$$
\begin{cases}S_{k}\left(D^{2} u\right)=g(x, u) & \text { in } \Omega, \\ u=0 & \text { on } \partial \Omega,\end{cases}
$$

where $g$ is a positive function satisfying the growth condition

$$
g(x, u) \leq f(x)+\sigma|u|^{p},
$$

where $f>0$ is a "smooth" function, $\sigma>0,0<p \leq k$. We also consider the symmetrized problem

$$
\begin{cases}S_{k}\left(D^{2} v\right)=f^{\#}+\sigma|v|^{p} & \text { in } B_{R} \\ v=0 & \text { on } \partial B_{R},\end{cases}
$$

where $f^{\#}$ is the spherically decreasing rearrangement of $f$ and $B_{R}$ is the ball centered at the origin, having the same $k^{\text {th }}$ mean radius as $\Omega$ (see Section 2 for definitions). Our purpose is to compare solutions to problems $(1.3)$ and $(1.5)$.

Existence of solutions to 1.3 in $\Phi_{0}^{k}(\Omega)$ has been investigated by Caffarelli, Nirenberg and Spruck in [7], where they treat the case $f=f(x)$ and establish the classical solvability for the Dirichlet problem

$$
\begin{cases}S_{k}\left(D^{2} u\right)=f(x) & \text { in } \Omega \\ u=0 & \text { on } \partial \Omega\end{cases}
$$

if and only if $\partial \Omega$ satisfies

$$
S_{k-1}\left(k_{1}, \ldots, k_{n-1}\right)>c_{0}>0
$$

where $k_{j}$ denotes the principle curvatures of $\partial \Omega$, oriented so that convex domains have nonnegative curvatures. Many other authors have been interested in the problem of existence and regularity of classical or viscosity solutions to $(1.3)$ under suitable hypotheses (see e.g. [20], [25], [26]). In any case existence and uniqueness of classical solutions to (1.3) are strictly related to the definition of the first eigenvalue of the Hessian operator $S_{k}\left(D^{2} u\right)$. In [13] and [24] for the case $k=n$ and in [26] for the general case $1 \leq k \leq n$, it is shown that there exists a positive constant $\lambda_{1}$, which depends only on $k, n$ and $\Omega$, such that: (i) the problem

$$
\begin{cases}S_{k}\left(D^{2} u\right)=|\lambda u|^{k} & \text { in } \Omega, \\ u=0 & \text { on } \partial \Omega,\end{cases}
$$

has a negative solution $\psi_{1} \in C^{\infty}(\Omega) \cap C^{1,1}(\bar{\Omega})$ for $\lambda=\lambda_{1}$; (ii) if $\left(\lambda^{\star}, \psi^{\star}\right) \in[0, \infty[\times$ $\left(C^{\infty}(\Omega) \cap C^{1,1}(\bar{\Omega})\right)$ is another solution to 1.6 , then $\lambda^{\star}=\lambda_{1}, \psi^{\star}=\alpha \psi_{1}$ for some positive constant $\alpha$. All these features suggest the well-known properties of the first eigenvalue 
of linear second order elliptic operators. For this reason we refer to $\lambda_{1}$ as the first eigenvalue of the Hessian operator. Moreover, the following variational characterization holds true (see [26]):

$$
\left(\lambda_{1}(\Omega)\right)^{k}=\inf \left\{-\int_{\Omega} u S_{k}\left(D^{2} u\right) d x: u \in \Phi_{0}^{k}(\Omega),\|u\|_{L^{k+1}(\Omega)}=1\right\} .
$$

Concerning uniqueness results for solutions to problem [1.3] we refer to [13], [24], [25], [26]. No attempt has been made here to investigate existence or regularity questions for problem 1.3 . Our aim is to prove comparison results by means of symmetrization techniques in the framework of papers [17], [4] in the case $k=n=2$, and [22], [23] in the general case $1 \leq k \leq n$. In [17], [22], [23] the case $\sigma=0$ is considered, while in [4] the case $\sigma>0$ has been investigated when $k=n=2$. Here we assume $\sigma>0$ and $1 \leq k \leq n$. The paper is organized as follows. After introducing notation in Section 2, in Section 3 we state our main result and we prove a comparison result between a suitable rearrangement of the solution to 1.3 , with the solution to the symmetrized problem (1.5). The rearrangements involved are those which preserve the so called "quermassintegrals" of level sets of solutions (see [17], [22], [23]) and the main tools are the AlexandrovFenchel inequalities (see Section 2). In the last section we prove some kind of Moser inequality related to Hessian integrals in the framework of papers by Moser [14] and Trudinger [19], [22].

\section{Notation and preliminaries}

Given a measurable function $u: \Omega \rightarrow \mathbb{R}$, we recall the definition of decreasing rearrangement of $u$. After defining the distribution function of $u$ by

$$
\mu(t)=\mathcal{L}^{n}(\{x \in \Omega:|u(x)|>t\}), \quad t \geq 0,
$$

where $\mathcal{L}^{n}$ denotes the $n$-dimensional Lebesgue measure in $\mathbb{R}^{n}$, the decreasing rearrangement of $u$ is the distribution function of $\mu$, i.e.

$$
u^{*}(s)=\sup \{t \geq 0: \mu(t) \geq s\}, \quad s \in[0,|\Omega|] .
$$

The following properties will prove useful in Section 3:

(i) $\|u\|_{L^{p}(\Omega)}=\left\|u^{*}\right\|_{L^{p}(0,|\Omega|)}, p \geq 1$;

(ii) (Hardy-Littlewood theorem) if $u, v: \Omega \rightarrow \mathbb{R}$ are measurable functions, then

$$
\int_{\Omega}|u v| d x \leq \int_{0}^{|\Omega|} u^{*}(s) v^{*}(s) d s
$$

By the spherically decreasing rearrangement of $u$ we mean

$$
u^{\#}(x)=u^{*}\left(\omega_{n}|x|^{n}\right), \quad x \in \Omega^{\#},
$$


where $\omega_{n}$ denotes the measure of the unit ball of $\mathbb{R}^{n}$ and $\Omega^{\#}$ is the ball centered at the origin, having the same measure as $\Omega$ (for more details see [18] and the references given there).

We go on with an appropriate definition of quermassintegral for non-convex domains (see [22]). For the basic theory of convex sets, the reader is referred, for example, to [6], [16]. Let $\Omega$ be a bounded domain in $\mathbb{R}^{n}$ with boundary $\partial \Omega \in C^{2}$, having principal curvatures $k^{\prime}=\left(k_{1}, \ldots, k_{n-1}\right)$ (oriented so that convex domains have non-negative curvatures). For $k=1, \ldots, n-1$ we define the $k^{\text {th }}$ mean curvature of $\partial \Omega$ by

$$
H_{k}(\partial \Omega)=S_{k}\left(k_{1}, \ldots, k_{n-1}\right),
$$

while

$$
H_{0}=S_{0} \equiv 1 \text {. }
$$

For $k=0, \ldots, n-1$ the quermassintegral $V_{k}(\Omega)$ is then defined by

$$
V_{k}(\Omega)=\frac{1}{n\left(\begin{array}{c}
n-1 \\
k
\end{array}\right)} \int_{\partial \Omega} H_{n-k-1}(\partial \Omega) d \mathcal{H}^{n-1},
$$

where $\mathcal{H}^{n-1}$ denotes the $(n-1)$-dimensional Hausdorff measure in $\mathbb{R}^{n}$. For $k=n$ we take $V_{n}(\Omega)=\mathcal{L}^{n}(\Omega)$. When $k \leq n-2$, we restrict attention to quermassintegrals $V_{k}(\Omega)$ of domains $\Omega$ which are $(n-k-1)$-convex, that is

$$
H_{j}(\partial \Omega) \geq 0, \quad j=1, \ldots, n-k-1,
$$

thereby ensuring that the integrand in $(2.1)$ is non-negative. When $\partial \Omega$ is connected, 2.2 is equivalent to $H_{n-k-1}(\partial \Omega) \geq 0$, while for $k=0$ we have to assume that $\Omega$ is $(n-1)$ convex, that is, the components of $\Omega$ are convex in the usual sense. We explicitly mention that in the latter case the following equality holds:

$$
V_{0}(\Omega)=\omega_{n} \times \text { number of components of } \Omega .
$$

When $k=n$, we clearly have $V_{n}\left(\Omega_{1}\right) \leq V_{n}\left(\Omega_{2}\right)$ whenever $\Omega_{1} \subset \Omega_{2}$. For convex domains we also have $V_{k}\left(\Omega_{1}\right) \leq V_{k}\left(\Omega_{2}\right)$ whenever $\Omega_{1} \subset \Omega_{2}$. But this property is not true in general when $\Omega_{1}$ and $\Omega_{2}$ are simply $(n-k-1)$-convex. We get around this difficulty by only considering functions belonging to $\Phi_{0}^{k}(\Omega)$, whose sublevel sets have the monotonicity property. Indeed, first of all, it is easy to see that, if $k>0$ and $u \in \Phi_{0}^{k}(\Omega)$, then $u$ is subharmonic and thus $u \leq 0$ in $\Omega$; so let $\Omega_{t}=\{x \in \Omega: u(x)<t\}$ and $\Omega_{s}=\{x \in \Omega: u(x)<s\}$, with $\min _{\Omega} u<s \leq t<0$. If $\Sigma_{s}=\{x \in \Omega: u(x)=s\}$ and $\Sigma_{t}=\{x \in \Omega: u(x)=t\}$ are non-degenerate level surfaces with $s \leq t$, then (see [22])

$$
V_{n-k+1}\left(\Omega_{s}\right) \leq V_{n-k+1}\left(\Omega_{t}\right) .
$$

One can also show a derivation formula for quermassintegrals of sublevel sets of a function $u \in \Phi_{0}^{k}(\Omega)$ (see [15]):

$$
\frac{d}{d t} V_{n-k+1}\left(\Omega_{t}\right)=\frac{1}{\left(\begin{array}{c}
n \\
k
\end{array}\right)} \int_{\Sigma_{t}} H_{k-1}\left(\Sigma_{t}\right)|D u|^{-1} d \mathcal{H}^{n-1} .
$$


Remark 2.1. Actually, 2.3 and 2.4 are still valid in a set wider than $\Phi_{0}^{k}(\Omega)$ (see [22]).

Now we introduce another useful notion. Let $\Omega$ be an $(n-k-1)$-convex domain with $k \leq n-2$. We define the $k^{\text {th }}$ mean radius of $\Omega$ by

$$
\zeta_{k}(\Omega)=\left(V_{k}(\Omega) / \omega_{n}\right)^{1 / k}, \quad k=1, \ldots, n, \quad \zeta_{k}(\emptyset)=0 .
$$

The isoperimetric inequalities, or Alexandrov-Fenchel inequalities, for quermassintegrals contained in [21] provide

$$
\zeta_{l}(\Omega) \leq \zeta_{k}(\Omega), \quad 1 \leq k \leq l \leq n .
$$

We note that 2.5 includes the classical isoperimetric inequality when $l=n$ and $k=$ $n-1$.

Now we are able to symmetrize a function. Let $u \in \Phi_{0}^{k}(\Omega), k=1, \ldots, n$; the $k$ symmetrand $u_{k-1}^{*}$ of $u$ is defined by

$$
u_{k-1}^{*}(x)=\sup \left\{t \leq 0: \zeta_{n-k+1}\left(\Omega_{t}\right) \leq|x|, D u \neq 0 \text { on } \Sigma_{t}\right\}
$$

for $|x| \leq R=\zeta_{n-k+1}(\Omega)$.

The following statements hold (see [22], [23]):

(1) set $\tilde{u}_{k-1}(r)=u_{k-1}^{*}(|x|)$ for $r=|x|$; then $\tilde{u}_{k-1}(0)=\min _{\Omega} u$ and $\tilde{u}_{k-1}(R)=0$;

(2) $\tilde{u}_{k-1}$ is a non-decreasing function on $[0, R]$;

(3) $\tilde{u}_{k-1} \in C^{0,1}([0, R])$ and moreover

$$
0 \leq \tilde{u}_{k-1}^{\prime} \leq \sup _{\Omega}|D u| \quad \text { a.e. }
$$

Remark 2.2. For $k=1$ we find $u_{0}^{*}(x)=u^{\#}(x)$, the Schwarz symmetrand of $u$. For $k=n=2$ we find $u_{1}^{*}(x)=u^{\star}(2 \pi|x|)$, where $u^{\star}$ is the rearrangement of $u$ with respect to the perimeter of its level sets (see [17]).

By definition, through 2.5 , it follows that $\zeta_{k}\left(\Omega_{t}\right) \leq \zeta_{k}\left(\left\{u_{m}^{*}<t\right\}\right)$ for $k \geq n-m$, and equality holds when $n-m=k$; this implies

$$
\|u\|_{L^{p}(\Omega)} \leq\left\|u_{k}^{*}\right\|_{L^{p}\left(B_{R}\right)}, \quad p \geq 1 .
$$

Let $u \in \Phi_{0}^{k}(\Omega)$ and let $\min _{\Omega}<t<0$. We recall the following Reilly equality (see [15]):

$$
\int_{\Omega_{t}} S_{k}\left(D^{2} u\right) d x=\frac{1}{k} \int_{\Sigma_{t}}|D u|^{k} H_{k-1}\left(\Sigma_{t}\right) d \mathcal{H}^{n-1} .
$$

Finally, we consider the following functional, known as the Hessian integral:

$$
I_{k, p}[u, \Omega]=\int_{m}^{0} d t \int_{\Sigma_{t}} H_{k-1}\left(\Sigma_{t}\right)|D u|^{p-1} d \mathcal{H}^{n-1} ;
$$

the following Pólya-Szegö principle holds (see [22]):

$$
I_{k, p}[u, \Omega] \geq I_{k, p}\left[u_{k-1}^{*}, B_{R}\right], \quad p \geq 1 .
$$


In the radial case the Hessian integral can be written as follows:

$$
\left.I_{k, p}\left(u_{k-1}^{*}, B_{R}\right)\right)=n\left(\begin{array}{l}
n-1 \\
k-1
\end{array}\right) \omega_{n} \int_{0}^{R} f^{p}\left(\omega_{n} \rho^{n-k+1}\right) \rho^{n-k} d \rho
$$

where $f\left(\omega_{n}|x|^{n-k+1}\right)=\left|\nabla u_{k-1}^{*}(x)\right|$.

\section{Comparison results}

In this section we will prove that the rearranged solution to problem 1.3 can be estimated by the solution to the conveniently symmetrized problem 1.5 . In order to prove this comparison result we will adapt an argument which appears in [9] in the case of the $p$-laplacian operator and in [4] in the case $k=n=2$.

Remark 3.1. Under the assumption that $\Omega$ is strictly $(k-1)$-convex, in [8] it is shown that, if $\Omega \in C^{3,1}, f$ is a smooth positive function in $\Omega$ and $\sigma<\left(\lambda_{1}(\Omega)\right)^{k}$ when $p=k$, then problem 1.3 has a solution in $C^{3, \alpha}(\Omega) \cap C(\bar{\Omega})$ for some $\alpha \in(0,1)$. In fact, other results concerning the existence of classical or non-classical solution to 1.3 may be found, for instance, in [11], [10], [24], [25], [26]. For uniqueness of solutions to [1.3] we refer to [26] for the case $p<1$ and to [25] for the case $1 \leq p \leq k$, provided $\Omega$ is sufficiently narrow or $\sigma$ sufficiently small.

Remark 3.2. We now turn our attention to the radial case. Let $u(x)=\phi(r),|x|=r$, be a radially symmetric function defined in the ball $B_{R}$. We have

$$
\begin{aligned}
& u_{x_{i}}=\phi^{\prime} \frac{x_{i}}{r} \\
& u_{x_{i} x_{i}}=\phi^{\prime \prime} \frac{x_{i}^{2}}{r^{2}}+\phi^{\prime} \frac{r^{2}-x_{i}^{2}}{r^{3}}, \quad u_{x_{i} x_{j}}=\phi^{\prime \prime} \frac{x_{i} x_{j}}{r^{2}}-\phi^{\prime} \frac{x_{i} x_{j}}{r^{3}}, \quad i \neq j,
\end{aligned}
$$

for $i, j=1, \ldots, n$. At the point $x=(r, 0, \ldots, 0), D^{2} u$ is diagonal, so $u_{x_{1} x_{1}}=\phi^{\prime \prime}$ and $u_{x_{j} x_{j}}=\phi^{\prime} / r, j \geq 2$. Since $S_{k}$ is invariant under rotations,

$$
\begin{aligned}
S_{k}\left(D^{2} u\right) & =\frac{(n-1) !}{(n-k) !(k-1) !} \phi^{\prime \prime}\left(\frac{\phi^{\prime}}{r}\right)^{k-1}+\frac{(n-1) !}{(n-k-1) ! k !}\left(\frac{\phi^{\prime}}{r}\right)^{k} \\
& =\frac{(n-1) !}{(n-k) !(k-1) !} r^{-n+1}\left(\frac{r^{n-k}}{k}\left(\phi^{\prime}\right)^{k}\right)^{\prime} .
\end{aligned}
$$

If $f(x)=\psi(r)$ is also radially symmetric, then 1.5 becomes an ordinary differential equation on $[0, R]$, with limit conditions $\phi^{\prime}(0)=\phi(R)=0$, i.e.

$$
\left\{\begin{array}{l}
\frac{(n-1) !}{(n-k) !(k-1) !} r^{-n+1}\left(\frac{r^{n-k}}{k}\left(\phi^{\prime}\right)^{k}\right)^{\prime}=f^{\#}+\sigma|\phi|^{p} \quad \text { in }(0, R), \\
\phi^{\prime}(0)=\phi(R)=0, \\
\left(\phi^{\prime \prime}, \phi^{\prime} / r, \ldots, \phi^{\prime} / r\right) \in \Gamma_{k} .
\end{array}\right.
$$


The existence (and uniqueness) of a solution to such a problem can be proved following the lines of [5], [3]. Furthermore, if $\left(\phi^{\prime \prime}, \phi^{\prime} / r, \ldots, \phi^{\prime} / r\right) \in \Gamma_{k}$, then $S_{1}\left(D^{2} \phi\right)=$ $r^{-n+1}\left(r^{n-1} \phi^{\prime}\right)^{\prime}>0$. Thus $G(r)=r^{n-1} \phi^{\prime}$ is an increasing function in $(0, R)$; since $G(0)=0$, we deduce that $G \geq 0$ and hence $\phi$ is increasing in $(0, R)$.

Theorem 3.1. Suppose that $f, g$ are smooth functions defined on a $(k-1)$-convex domain $\Omega$ in $\mathbb{R}^{n}$ such that $(1.4)$ is satisfied, and let $u, v$ be classical solutions to problems 1.3 and 1.5 respectively, with $\sigma<\min \left\{\left(\lambda_{1}(\Omega)\right)^{k},\left(\lambda_{1}\left(B_{R}\right)\right)^{k}\right\}$ when $p=k$, and $\sigma$ sufficiently small when $0<p<k$. Then

$$
0 \geq u_{k-1}^{*}(x) \geq v(x), \quad x \in B_{R} .
$$

Proof. Let $\min _{\Omega} u<t<0$ and let us integrate the equation in 1.3 on the sublevel set $\Omega_{t} ;$ then

$$
\int_{\Omega_{t}} S_{k}\left(D^{2} u\right) d x \leq \int_{\Omega_{t}} f d x+\sigma \int_{\Omega_{t}}(-u)^{p} d x
$$

By (2.8), the Hölder inequality and (2.4) we get

$$
\begin{aligned}
\int_{\Omega_{t}} S_{k}\left(D^{2} u\right) d x & =\frac{1}{k} \int_{\Sigma_{t}}|D u|^{k} H_{k-1}\left(\Sigma_{t}\right) d \mathcal{H}^{n-1} \geq \frac{1}{k} \frac{\left(\int_{\Sigma_{t}} H_{k-1}\left(\Sigma_{t}\right) d \mathcal{H}^{n-1}\right)^{k+1}}{\left(\int_{\Sigma_{t}} H_{k-1}\left(\Sigma_{t}\right)|D u|^{-1} d \mathcal{H}^{n-1}\right)^{k}} \\
& =\frac{1}{k} \frac{\left(\int_{\Sigma_{t}} H_{k-1}\left(\Sigma_{t}\right) d \mathcal{H}^{n-1}\right)^{k+1}}{\left(\begin{array}{l}
n \\
k
\end{array}\right)^{k}\left(\frac{d}{d t} V_{n-k+1}\left(\Omega_{t}\right)\right)^{k}} .
\end{aligned}
$$

We recall that, by definition,

$$
\int_{\Sigma_{t}} H_{k-1}\left(\Sigma_{t}\right) d \mathcal{H}^{n-1}=n\left(\begin{array}{l}
n-1 \\
n-k
\end{array}\right) V_{n-k}\left(\Omega_{t}\right)=n\left(\begin{array}{l}
n-1 \\
n-k
\end{array}\right) \omega_{n}\left(\zeta_{n-k}\left(\Omega_{t}\right)\right)^{(n-k)(k+1)},
$$

so we can write

$$
\int_{\Omega_{t}} S_{k}\left(D^{2} u\right) d x \geq \frac{n^{k+1}\left(\begin{array}{l}
n-1 \\
n-k
\end{array}\right)^{k+1} \omega_{n}^{k+1}}{k\left(\begin{array}{l}
n \\
k
\end{array}\right)^{k}} \frac{\left(\zeta_{n-k}\left(\Omega_{t}\right)\right)^{(n-k)(k+1)}}{\left(\frac{d}{d t} V_{n-k+1}\left(\Omega_{t}\right)\right)^{k}} .
$$

On the other hand, by the definition of the $k^{\text {th }}$ mean radius we have

$$
\frac{d}{d t} V_{n-k+1}\left(\Omega_{t}\right)=\frac{\omega_{n}}{n-k+1} \zeta_{n-k+1}^{n-k}\left(\Omega_{t}\right) \frac{d}{d t} \zeta_{n-k+1}\left(\Omega_{t}\right)
$$

thus, recalling the isoperimetric inequalities 2.5, we obtain

$$
\int_{\Omega_{t}} S_{k}\left(D^{2} u\right) d x \geq \frac{n^{k+1}}{k \omega_{n}^{k-1}} \frac{\left(\begin{array}{l}
n-1 \\
n-k
\end{array}\right)^{k+1}}{\left(\begin{array}{l}
n \\
k
\end{array}\right)^{k}}(n-k+1)^{k} \frac{\zeta_{n-k+1}^{n-k}\left(\Omega_{t}\right)}{\left(\frac{d}{d t} \zeta_{n-k+1}\left(\Omega_{t}\right)\right)^{k}}
$$


Let us come back to (3.2). For the first integral on the right hand side of (3.2), by the Hardy-Littlewood inequality and (2.5), we obtain

$$
\int_{\Omega_{t}} f d x \leq \int_{0}^{\mu(t)} f^{*}(s) d s \leq \int_{0}^{\omega_{n} \zeta_{n-k+1}^{n}\left(\Omega_{t}\right)} f^{*}(s) d s
$$

while for the second one we get

$$
\begin{aligned}
\int_{\Omega_{t}}(-u)^{p} d x & =\int_{-\infty}^{t}(-s)^{p} \mu^{\prime}(s) d s=(-t)^{p} \mu(t)+p \int_{-\infty}^{t}(-s)^{p-1} \mu(s) d s \leq \\
& \leq \omega_{n}(-t)^{p} \zeta_{n-k+1}^{n}\left(\Omega_{t}\right)+\omega_{n} p \int_{-\infty}^{t}(-s)^{p-1} \zeta_{n-k+1}^{n}\left(\Omega_{s}\right) d s
\end{aligned}
$$

which, in terms of rearrangements, becomes

$$
\begin{aligned}
\int_{\Omega_{t}}(-u)^{p} d x & \leq \omega_{n} r^{n}\left(-\tilde{u}_{k-1}(r)\right)^{p}+\omega_{n} p \int_{0}^{r} s^{n}\left(-\tilde{u}_{k-1}(s)\right)^{p-1} \tilde{u}_{k-1}(s)^{\prime} d s \\
& =n \omega_{n} \int_{0}^{r} s^{n-1}\left(-\tilde{u}_{k-1}(s)\right)^{p} d s,
\end{aligned}
$$

with $r=\zeta_{n-k+1}\left(\Omega_{t}\right)$. Observing that

$$
\frac{\zeta_{n-k+1}^{n-k}\left(\Omega_{t}\right)}{\left(\frac{d}{d t} \zeta_{n-k+1}\left(\Omega_{t}\right)\right)^{k}}=r^{n-k}\left(\tilde{u}_{k-1}(r)^{\prime}\right)^{k}
$$

by $3.3-3.5$ we get

$$
c_{n, k} r^{n-k}\left(\tilde{u}_{k-1}(r)^{\prime}\right)^{k} \leq \int_{0}^{\omega_{n} r^{n}} f^{*}(s) d s+n \omega_{n} \sigma \int_{0}^{r} s^{n-1}\left(-\tilde{u}_{k-1}(s)\right)^{p} d s
$$

where

$$
c_{n, k}=\frac{n^{k+1}}{k \omega_{n}^{k-1}} \frac{\left(\begin{array}{c}
n-1 \\
n-k
\end{array}\right)^{k+1}}{\left(\begin{array}{l}
n \\
k
\end{array}\right)^{k}}(n-k+1)^{k} .
$$

Obviously the radial solution $v$ to the symmetrized problem (1.5) satisfies

$$
c_{n, k} r^{n-k}\left(\tilde{v}_{k-1}(r)^{\prime}\right)^{k}=\int_{0}^{\omega_{n} r^{n}} f^{*}(s) d s+n \omega_{n} \sigma \int_{0}^{r} s^{n-1}\left(-\tilde{v}_{k-1}(s)\right)^{p} d s .
$$

Let $U(r)=\int_{0}^{r} s^{n-1} \tilde{u}_{k-1}(s)^{p} d s$ and $V(r)=\int_{0}^{r} s^{n-1} \tilde{v}_{k-1}(s)^{p} d s$; let us prove that $U(r) \leq V(r)$ for all $r \in[0, R]$. Set

$$
\varphi_{1}(s)=\frac{\left(U(s)^{(p+1) / p}-V(s)^{(p+1) / p}\right)^{+}}{U(s)^{1 / p}} ;
$$


integrating by parts we obtain

$$
\begin{aligned}
\int_{U>V}\left(-\tilde{u}_{k-1}(r)^{\prime}\right) \varphi_{1}(r) d r & =\int_{U>V} \tilde{u}_{k-1}(r) \varphi_{1}^{\prime}(r) d r \\
=\int_{U>V} \tilde{u}_{k-1}(r)\left(r \tilde{u}_{k-1}(r)^{p}\right. & -\frac{p+1}{p}\left(\frac{V(r)}{U(r)}\right)^{1 / p} r \tilde{v}_{k-1}(r)^{p} \\
& \left.+\frac{1}{p}\left(\frac{V(r)}{U(r)}\right)^{(p+1) / p} r \tilde{u}_{k-1}(r)^{p}\right) d r .
\end{aligned}
$$

Analogously, by considering

$$
\varphi_{2}(s)=\frac{\left(U(s)^{(p+1) / p}-V(s)^{(p+1) / p}\right)^{+}}{V(s)^{1 / p}}
$$

and integrating by parts we have

$$
\begin{aligned}
\int_{U>V}\left(-\tilde{v}_{k-1}(r)^{\prime}\right) \varphi_{2}(r) d r & =\int_{U>V} \tilde{v}_{k-1}(r) \varphi_{2}^{\prime}(r) d r \\
=\int_{U>V} \tilde{v}_{k-1}(r)\left(-r \tilde{v}_{k-1}(r)^{p}\right. & +\frac{p+1}{p}\left(\frac{U(r)}{V(r)}\right)^{1 / p} r \tilde{v}_{k-1}(r)^{p} \\
& \left.-\frac{1}{p}\left(\frac{U(r)}{V(r)}\right)^{(p+1) / p} r \tilde{v}_{k-1}(r)^{p}\right) d r .
\end{aligned}
$$

Let $\gamma=(p+1) / p$; subtracting 3.9 from 3.8 we get

$$
\begin{aligned}
& \int_{U>V}\left[\left(-\tilde{u}_{k-1}(r)^{\prime}\right) \varphi_{1}(r)+\tilde{v}_{k-1}(r)^{\prime} \varphi_{2}(r)\right] d r= \\
& \int_{U>V} r U^{\gamma}\left[\left(\frac{\tilde{u}_{k-1}^{1 /(\gamma-1)}}{U}\right)^{\gamma}-\left(\frac{\tilde{v}_{k-1}^{1 /(\gamma-1)}}{V}\right)^{\gamma}-\gamma\left(\frac{\tilde{v}_{k-1}^{1 /(\gamma-1)}}{V}\right)^{\gamma-1}\left(\frac{\tilde{u}_{k-1}^{1 /(\gamma-1)}}{U}-\frac{\tilde{v}_{k-1}^{1 /(\gamma-1)}}{V}\right)\right] \\
& +\int_{U>V} r V^{\gamma}\left[\left(\frac{\tilde{v}_{k-1}^{1 /(\gamma-1)}}{V}\right)^{\gamma}-\left(\frac{\tilde{u}_{k-1}^{1 /(\gamma-1)}}{U}\right)^{\gamma}-\gamma\left(\frac{\tilde{u}_{k-1}^{1 /(\gamma-1)}}{U}\right)^{\gamma-1}\left(\frac{\tilde{v}_{k-1}^{1 /(\gamma-1)}}{V}-\frac{\tilde{u}_{k-1}^{1 /(\gamma-1)}}{U}\right)\right] \\
& =I_{1}+I_{2} .
\end{aligned}
$$

Both $I_{1}$ and $I_{2}$ are non-negative since the function $g(t)=t^{\gamma}$ is convex for all $\gamma>1$. On the other hand, by 3.6) and 3.7,

$$
\begin{aligned}
I_{1}+I_{2} \leq \int_{U>V} & \left(\frac{U(r)^{(p+1) / p}-V(r)^{(p+1) / p}}{r^{(n-k) / k}}\right) \\
\times & {\left[\left(\frac{c_{1} F(r)+c_{2} U(r)}{U(r)^{k / p}}\right)^{1 / k}-\left(\frac{c_{1} F(r)+c_{2} V(r)}{V(r)^{k / p}}\right)^{1 / k}\right] d r, }
\end{aligned}
$$

where $F(s)=\int_{0}^{\omega_{n} s^{n}} f^{*}(r) d r, c_{1}=1 / c_{n, k}, c_{2}=n \omega_{n} / c_{n, k}$. The right hand side of 3.10 is always negative, since the function $h(t)=(1+t) / t^{k / p}$ is decreasing whenever $p \leq k$. 
It follows that $|U>V|=0$, i.e. $U(s) \leq V(s)$ for all $s \in(0, R)$. This estimate, combined with (3.6) and 3.7), gives (3.1) and the claim follows.

Remark 3.3. We recall that $V_{0}(\Omega)=\omega_{n} \times$ number of components of $\Omega$. Since a $C^{2}$ domain is $(n-1)$-convex if and only if it is convex (see [21]), if $u$ is a classical solution to 1.3 for $k=n$ and $\lambda\left(D^{2} u\right) \in \Gamma_{n}$, its level sets $\Omega_{t}$ are convex for a.e. $t$ and this implies $V_{0}\left(\Omega_{t}\right)=\omega_{n}$ for a.e. $t$.

Remark 3.4. It immediately follows from (3.1) and 2.7) that

$$
\|u\|_{L^{p}(\Omega)} \leq\|v\|_{L^{p}\left(B_{R}\right)}, \quad p \geq 1 .
$$

Proposition 3.1. Under the assumptions of Theorem 3.1 we have

$$
I_{k, k+1}(u, \Omega) \leq I_{k, k+1}\left(v, B_{R}\right) .
$$

Proof. The above estimate is an easy consequence of the inequality

$$
\begin{aligned}
\int_{\Sigma_{t}}|D u|^{k} H_{k-1}\left(\Sigma_{t}\right) d \mathcal{H}^{n-1} \leq & k \int_{0}^{\omega_{n} \zeta_{n-k+1}\left(\Omega_{t}\right)} f^{*}(r) d r \\
& +n \omega_{n} k \int_{0}^{\zeta_{n-k+1}\left(\Omega_{t}\right)} s^{n-1}\left(-\tilde{u}_{k-1}(s)\right)^{p} d s
\end{aligned}
$$

and the equality holding for $v$.

\section{Moser type inequalities for Hessian integrals in the limit case $p=n-k+1$}

In [22] Trudinger has proved some kind of Sobolev inequalities for functions in $A_{k-1}(\Omega)$. Namely, if $\Omega$ is a $(k-1)$-convex subset of $\mathbb{R}^{n}, k=1, \ldots, n$, and $u \in \Phi_{0}^{k}(\Omega)$, then

$$
\|u\|_{L^{q}(\Omega)}^{p} \leq C I_{k, p}(u, \Omega)
$$

for any $q \leq n p /(n-k+1-p)$ if $p<n-k+1$, and for $q \leq+\infty$ if $p>n-k+1$, with a constant $C$ depending on $k, n, p$ and $\Omega$. In the limit case $p=n-k+1$, he has obtained the estimate

$$
\|u\|_{L_{\Psi}(\Omega)}^{p} \leq C I_{k, p}(u, \Omega)
$$

where $L_{\Psi}(\Omega)$ is the Orlicz space associated to the function

$$
\Psi(t)=e^{|t|^{p^{\prime}}}-1 .
$$

This last result recovers the traditional one when $k=1$ (see [19]); in this case it is well known that the estimate (4.1) has been made more precise by many authors in many directions (see, e.g., [14], [1], [2]). For example, in [14] it is proved that if $u \in C_{0}^{\infty}(\Omega)$ and $\|\nabla u\|_{L^{n}(\Omega)} \leq 1$, then

$$
\int_{\Omega} e^{\beta|u(x)|^{n^{\prime}}} d x \leq C(n)|\Omega|, \quad \forall \beta \leq \beta_{n}=\left(n \omega_{n}^{1 / n}\right)^{n^{\prime}} .
$$


As a matter of fact the integral in 4.2$\}$ is finite for any $\beta$, but it is uniformly bounded only for $\beta \leq \beta_{n}=\left(n \omega_{n}^{1 / n}\right)^{n^{\prime}}$. Indeed, if $\beta_{n}=\left(n \omega_{n}^{1 / n}\right)^{n^{\prime}}$, then a sequence $\left\{u_{m}\right\} \subset C_{0}^{\infty}(\Omega)$ can be constructed so that $\left\|\nabla u_{m}\right\|_{L^{n}(\Omega)} \leq 1$ and

$$
\lim _{m \rightarrow+\infty} \int_{\Omega} e^{\beta\left|u_{m}(x)\right|^{n^{\prime}}} d x=+\infty .
$$

Similar questions arise concerning estimate 4.1 . We will prove the following

Theorem 4.1. Let $\Omega$ be a $(k-1)$-convex subset of $\mathbb{R}^{n}, k=1, \ldots, n$, and let $u \in \Phi_{0}^{k}(\Omega)$ be such that

$$
J_{k, p}(u, \Omega)=\frac{I_{k, p}(u, \Omega)}{\left(\begin{array}{l}
n-1 \\
k-1
\end{array}\right)} \leq 1 \quad \text { for } p=n-k+1 .
$$

Then

$$
\int_{\Omega} e^{\beta|u(x)|^{(n-k+1) /(n-k)}} d x \leq c_{n, k}\left(V_{n-k+1}(\Omega)\right)^{n /(n-k+1)}
$$

for all $\beta \leq \beta_{n, k}=\left(n \omega_{n}^{1 /(n-k+1)}\right)^{(n-k+1) /(n-k)}$.

In order to prove the above theorem we need the following lemma due to Adams [1].

Lemma 4.1. Let $a(s, t)$ be a non-negative measurable function on $\mathbb{R} \times[0,+\infty[$ such that, for some $p \in] 1,+\infty[$,

$$
\begin{aligned}
& a(s, t) \leq 1 \quad \text { for a.e. } 0<s<t \\
& \sup _{t>0}\left(\int_{-\infty}^{0} a(s, t)^{p^{\prime}} d s+\int_{0}^{+\infty} a(s, t)^{p^{\prime}} d s\right)^{1 / p^{\prime}}=\gamma<+\infty
\end{aligned}
$$

Suppose that a non-negative function $F(s)$ on $\mathbb{R}$ satisfies

$$
\int_{-\infty}^{+\infty} F(s)^{p} d s \leq 1
$$

Then there exists a constant $c_{0}=c_{0}(p, \gamma)$ such that

$$
\int_{0}^{+\infty} e^{-G(t)} d t \leq c_{0}, \quad \text { where } G(t)=t-\left(\int_{-\infty}^{+\infty} a(s, t) F(s) d s\right)^{p^{\prime}} .
$$

Proof of Theorem 4.1. Let us first observe that by the Pólya-Szegő principle (2.9) and by 2.7) we have

$$
\begin{aligned}
J_{k, p}\left(u_{k-1}^{*}, B_{R}\right) & \leq J_{k, p}(u, \Omega), \\
\int_{\Omega} \exp \left(\beta|u|^{(n-k+1) /(n-k)}\right) d x & \leq \int_{B_{R}} \exp \left(\beta\left|u_{k-1}^{*}\right|^{(n-k+1) /(n-k)}\right) d x ;
\end{aligned}
$$

then it is enough to prove the statement for $u(x)=u_{k-1}^{*}(x)$. Set

$$
f\left(\omega_{n}|x|^{n-k+1}\right)=\left|\nabla u_{k-1}^{*}(x)\right|
$$


this implies that $f(s)=\omega_{n}(n-k+1) \tilde{u}_{k-1}^{\prime}(s)\left(s / \omega_{n}\right)^{(n-k) /(n-k+1)}$. We have

$$
-\tilde{u}_{k-1}(s)=\frac{1}{(n-k+1) \omega_{n}^{1 /(n-k+1)}} \int_{s}^{V_{n-k+1}(\Omega)} f(r) r^{1 /(n-k+1)} \frac{d r}{r} .
$$

It follows that

$$
\begin{aligned}
& -\tilde{u}_{k-1}\left(e^{-(n-k+1) t / n} V_{n-k+1}(\Omega)\right) \\
& =\frac{1}{n \omega_{n}^{1 /(n-k+1)}} \int_{0}^{t} f\left(e^{-(n-k+1) s / n} V_{n-k+1}(\Omega)\right)\left(e^{-(n-k+1) s / n} V_{n-k+1}(\Omega)\right)^{1 /(n-k+1)} d s \\
& =\frac{1}{n \omega_{n}^{1 /(n-k+1)}} \int_{-\infty}^{+\infty} a(s, t) F(s) d s,
\end{aligned}
$$

where

$$
\begin{aligned}
a(s, t) & = \begin{cases}0 & \text { if } s<0 \text { or } s>t, \\
1 & \text { if } 0 \leq s \leq t,\end{cases} \\
F(s) & = \begin{cases}f\left(e^{-(n-k+1) s / n} V_{n-k+1}(\Omega)\right)\left(e^{-(n-k+1) s / n} V_{n-k+1}(\Omega)\right)^{1 / p} & \text { if } s \geq 0, \\
0 & \text { if } s<0 .\end{cases}
\end{aligned}
$$

It is clear that 4.4 and 4.5) are satisfied. On the other hand, by 2.10,

$$
\begin{aligned}
J_{k, p}\left(u_{k-1}^{*}, B_{R}\right) & =\frac{I_{k, p}\left(u_{k-1}^{*}, B_{R}\right)}{\left(\begin{array}{l}
n-1 \\
k-1
\end{array}\right)}=n \omega_{n} \int_{0}^{R} f^{p}\left(\omega_{n} \rho^{n-k+1}\right) \rho^{n-k} d \rho \\
& =\frac{n}{n-k+1} \int_{0}^{V_{n-k+1}(\Omega)} f^{p}(r) d r \\
& =\int_{0}^{+\infty} f\left(e^{-(n-k+1) s / n} V_{n-k+1}(\Omega)\right)^{n-k+1} e^{-(n-k+1) s / n} V_{n-k+1}(\Omega) d s \\
& =\int_{0}^{+\infty} F(s)^{p} d s \leq 1 .
\end{aligned}
$$

Then Lemma 4.1 gives

$$
\int_{0}^{+\infty} \exp \left(-t+\left(\left|\tilde{u}_{k-1}\left(e^{-(n-k+1) t / n} V_{n-k+1}(\Omega)\right)\right| n \omega_{n}^{1 /(n-k+1)}\right)^{(n-k+1) /(n-k)}\right) d t \leq c_{0},
$$

that is,

$$
\begin{aligned}
& \int_{0}^{V_{n-k+1}(\Omega)} \exp \left(\left|\tilde{u}_{k-1}(s)\right| n \omega_{n}^{1 /(n-k+1)}\right)^{\frac{n-k+1}{n-k}} s^{\frac{k-1}{n-k+1}} d s \\
& \quad \leq c_{0}\left(\frac{n-k+1}{n}\right)\left(V_{n-k+1}(\Omega)\right)^{n /(n-k+1)}
\end{aligned}
$$

and the claim follows. 
Remark 4.1 (Case $k \leq n / 2$ ). As a matter of fact, by definition of $\Phi_{0}^{k}(\Omega)$, the integral in (4.3) is finite for every $\beta$, but it is uniformly bounded only for $\beta \leq \beta_{n, k}$. Let us show that, if $\beta>\beta_{n, k}$, then there exists a sequence $\left\{u_{m}\right\}_{m \in \mathbb{N}} \subset \Phi_{0}^{k}(\Omega)$ such that $J_{k, p}\left(u_{m}, \Omega\right) \leq 1$ and

$$
\lim _{n \rightarrow+\infty} \int_{\Omega} e^{\beta\left|u_{m}(x)\right|^{(n-k+1) /(n-k)}} d x=+\infty .
$$

Let $B_{R}$ be the ball centered at the origin such that $V_{n-k+1}(\Omega)=\omega_{n} R^{n-k+1}=1$ and define

$$
\begin{aligned}
& u_{m}(x) \\
& = \begin{cases}\frac{m^{-1 /(n-k+1)}}{n \omega_{n}^{1 /(n-k+1)}\left(\frac{n}{n-k+1}\right)^{(n-k) /(n-k+1)}} \log \left(\omega_{n}|x|^{n-k+1}\right) & \text { if } \omega_{n}|x|^{n-k+1}>e^{-m}, \\
a_{m}|x|^{3}+b_{m}|x|^{2}+d_{m} & \text { if } \omega_{n}|x|^{n-k+1} \leq e^{-m},\end{cases}
\end{aligned}
$$

where

$$
\begin{aligned}
a_{m}= & -\frac{2}{3} m^{-1 /(n-k+1)} \omega_{n}^{2 /(n-k+1)}\left(\frac{n}{n-k+1}\right)^{-1 /(n-k+1)} e^{3 m /(n-k+1)} ; \\
b_{m}= & \frac{3}{2} m^{-1 /(n-k+1)} \omega_{n}^{1 /(n-k+1)}\left(\frac{n}{n-k+1}\right)^{-1 /(n-k+1)} e^{2 m /(n-k+1)} ; \\
d_{m}= & -\frac{m^{(n-k) /(n-k+1)}}{n \omega_{n}^{1 /(n-k+1)}}\left(\frac{n}{n-k+1}\right)^{(n-k) /(n-k+1)} \\
& -\frac{5}{6} m^{-1 /(n-k+1)} \frac{n-k+1}{n \omega_{n}^{1 /(n-k+1)}}\left(\frac{n}{n-k+1}\right)^{(n-k) /(n-k+1)} .
\end{aligned}
$$

We explicitly mention that $u_{m} \in C^{2}\left(B_{R}\right)$ and $S_{j}\left(D^{2} u\right)>0$ for any $1 \leq j \leq k$ so that $u \in \Phi_{0}^{k}(\Omega)$. First of all we compute $J_{k, p}\left(u_{m}, B_{R}\right)$. We have

$$
\begin{aligned}
& f\left(\omega_{n}|x|^{n-k+1}\right)=\left|\nabla u_{m}(x)\right| \\
& \quad= \begin{cases}\frac{m^{-1 /(n-k+1)}}{n \omega_{n}^{1 /(n-k+1)}(n-k+1)\left(\frac{n}{n-k+1}\right)^{(n-k) /(n-k+1)}} & \frac{1}{|x|} \\
3 a_{m}|x|^{2}+2 b_{m}|x| & \text { if } \omega_{n}|x|^{n-k+1}>e^{-m},\end{cases}
\end{aligned}
$$

then, setting $r_{m}=\left(e^{-m} / \omega_{n}\right)^{1 /(n-k+1)}$, we have

$$
\begin{aligned}
I_{k, p}\left(u_{m}, B_{R}\right) & =n\left(\begin{array}{l}
n-1 \\
k-1
\end{array}\right) \omega_{n} \int_{0}^{R} f^{p}\left(\omega_{n} \rho^{n-k+1}\right) \rho^{n-k} d \rho \\
= & n\left(\begin{array}{l}
n-1 \\
k-1
\end{array}\right) \omega_{n}\left[\int_{0}^{r_{m}} f^{p}\left(\omega_{n} \rho^{n-k+1}\right) \rho^{n-k} d \rho+\int_{r_{m}}^{R} f^{p}\left(\omega_{n} \rho^{n-k+1}\right) \rho^{n-k} d \rho\right] \\
= & \frac{c(n, k)}{m}+\left(\begin{array}{l}
n-1 \\
k-1
\end{array}\right),
\end{aligned}
$$


where $c(n, k)$ is a positive constant depending only on $n$ and $k$. This implies

$$
\lim _{m \rightarrow+\infty} I_{k, p}\left(u_{m}, B_{R}\right)=\left(\begin{array}{l}
n-1 \\
k-1
\end{array}\right)
$$

and then

$$
\lim _{m \rightarrow+\infty} J_{k, p}\left(u_{m}, B_{R}\right)=1
$$

Furthermore, we have

$$
\begin{aligned}
\int_{B_{R}} e^{\beta\left|u_{m}(x)\right|^{(n-k+1) /(n-k)} d x} & \geq \int_{0}^{e^{-m}} \exp \left(\frac{m \beta}{\beta_{n, k}} \frac{n}{n-k+1}\right) s^{(k-1) /(n-k+1)} d s \\
& =\frac{n-k+1}{n} \exp \left[\frac{n}{n-k+1}\left(\frac{\beta}{\beta_{n, k}}-1\right) m\right],
\end{aligned}
$$

which tends to $+\infty$ as $m$ goes to $+\infty$ when $\beta>\beta_{n, k}$.

Remark 4.2. When $u \in C^{2}(\Omega)$ is a convex function, vanishing on the boundary $\partial \Omega$, estimate 4.3 can be improved in the sense that, if $J_{k, p}(u, \Omega) \leq 1$, then there exists a positive constant $C=C(n, k)$ such that $\|u\|_{L^{\infty}(\Omega)} \leq C$. In fact, in this case the $k$ symmetrand of $u, u_{k-1}^{*}$, is a radially symmetric, convex function in the ball $B_{R}$, vanishing on $\partial B_{R}$ and belonging to $C^{2}\left(B_{R}\right) \cap C\left(\bar{B}_{R}\right)$. By the Pólya-Szegô principle and 2.7 we know that

$$
\|u\|_{L^{\infty}(\Omega)} \leq\left\|u_{k-1}^{*}\right\|_{L^{\infty}\left(B_{R}\right)}, \quad I_{k, p}(u, \Omega) \geq I_{k, p}\left(u_{k-1}^{*}, B_{R}\right),
$$

so, once again, it is enough to consider $u_{k-1}^{*}$. Without loss of generality we suppose that $V_{n-k+1}(\Omega)=V_{n-k+1}\left(B_{R}\right)=1$; we obtain, recalling that $J_{k, p}(u, \Omega) \leq 1$,

$$
\begin{aligned}
\left\|u_{k-1}^{*}\right\|_{L^{\infty}\left(B_{R}\right)}=\left|u_{k-1}^{*}(0)\right|=\int_{0}^{1}\left(\tilde{u}_{k-1}(s)\right)^{\prime} d s \\
\quad \leq \int_{0}^{1} \frac{1}{1-s}\left(\int_{s}^{1}\left(\tilde{u}_{k-1}(r)\right)^{\prime} d r\right) d s=\int_{0}^{1}\left(\tilde{u}_{k-1}(s)\right)^{\prime}|\log (1-s)| d s \\
\quad \leq\left(\int_{0}^{1}\left(\tilde{u}_{k-1}(s)^{\prime}\right)^{n-k+1} s^{n-k} d s\right)^{\frac{1}{n-k+1}}\left(\int_{0}^{1} \frac{|\log (1-s)|^{(n-k+1) /(n-k)}}{s} d s\right)^{\frac{n-k}{n-k+1}} \\
\leq C(n, k) .
\end{aligned}
$$

\section{References}

[1] Adams, D. R.: A sharp inequality of J. Moser for higher order derivatives. Ann. of Math. 128, 385-398 (1988) Zbl 0672.31008 MR 0960950

[2] Alvino, A., Ferone, V., Trombetti, G.: Moser type inequalities in Lorentz spaces. Potential Anal. 5, 273-299 (1996) Zbl 0856.46020 MR 1389498

[3] Alvino, A., Ferone, V., Trombetti, G.: On properties of some nonlinear eigenvalues. SIAM J. Math. Anal. 29, 437-451 (1998) Zbl 0908.35094 MR 1616519 
[4] Brandolini, B., Trombetti, C.: A symmetrization result for Monge-Ampère type equations. Math. Nachr. 280, 467-478 (2007)

[5] Brezis, H., Oswald, L.: Remarks on sublinear elliptic equations. Nonlinear Anal. 10, 55-64 (1986) Zbl 0593.35045 MR 0820658

[6] Burago, Yu. D., Zalgaller, V. A.: Geometric Inequalities. Springer (1988) Zbl 0633.53002 MR 0936419

[7] Caffarelli, L., Nirenberg, L., Spruck, J.: The Dirichlet problem for nonlinear second order elliptic equations, III: Functions of the eigenvalues of the Hessian. Acta Math. 155, 261-301 (1985) Zbl 0654.35031 MR 0806416

[8] Chou, K.-S., Wang, X.-J.: A variational theory of the Hessian equation. Comm. Pure Appl. Math. 54, 1029-1064 (2001) Zbl 1035.35037 MR 1835381

[9] Ferone, V., Messano, B.: A symmetrization result for nonlinear elliptic equations. Rev. Mat. Complut. 17, 261-276 (2004) Zbl pre02109936 MR 2083955

[10] Geng, D., Yu, Q., Qu, C.: The eigenvalue problem for hessian operators. Nonlinear Anal. 25, 27-40 (1995) Zbl 0869.35072 MR 1331986

[11] Ivochkina, N. M.: Solution of the Dirichlet problem for the equation of curvature of order $m$. Dokl. Akad. Nauk SSSR 299, 35-38 (1988) (in Russian); English transl.: Soviet Math. Dokl. 37, 322-325 (1988) Zbl 0699.35096 MR 0936908

[12] Korevaar, N. J.: A priori interior gradient bounds for solutions to elliptic Weingarten equations. Ann. Inst. H. Poincaré Anal. Non Linéaire 4, 405-421 (1987) Zbl 0644.35041 MR 0921546

[13] Lions, P.-L.: Two remarks on Monge-Ampère equations. Ann. Mat. Pura Appl. 142, 263-275 (1985) Zbl 0594.35023 MR 0839040

[14] Moser, J.: A sharp form of an inequality by N. Trudinger. Indiana Univ. Math. J. 20, 10771092 (1971) Zbl 0213.13001 MR 0301504

[15] Reilly, R. C.: On the Hessian of a function and the curvatures of its graph. Michigan Math. J. 20, 373-383 (1973) Zbl 0267.53003 MR 0334045

[16] Santaló, L.: Integral Geometry and Geometric Probability. Addison-Wesley (1976) Zbl 0342.53049 MR 0433364

[17] Talenti, G.: Some estimates of solutions to Monge-Ampère equations in dimension two. Ann. Scuola Norm. Sup. Pisa (2) 7, 183-230 (1981) Zbl 0467.35044 MR 0623935

[18] Talenti, G.: Linear elliptic p.d.e.'s: level sets, rearrangements and a priori estimates of solutions. Boll. Un. Mat. Ital. B (6) 4, 917-949 (1985) Zbl 0602.35025 MR 0831299

[19] Trudinger, N. S.: On imbeddings into Orlicz spaces and some applications. J. Math. Mech. 17, 473-483 (1967) Zbl 0163.36402 MR 0216286

[20] Trudinger, N. S.: The Dirichlet problem for the prescribed curvature equations. Arch. Ration. Mech. Anal. 111, 153-180 (1990) Zbl 0721.35018 MR 1057653

[21] Trudinger, N.S.: Isoperimetric inequalities for quermassintegrals. Ann. Inst. H. Poincaré Anal. Non Linéaire 11, 411-425 (1994) Zbl 0859.52001 MR 1287239

[22] Trudinger, N. S.: On new isoperimetric inequalities and symmetrization. J. Reine Angew. Math. 488, 203-220 (1997) Zbl 0883.52006 MR 1465371

[23] Tso, K.: On symmetrization and hessian equations. J. Anal. Math. 52, 94-106 (1989) Zbl 0675.35040 MR 0981497

[24] Tso, K.: On a real Monge-Ampère functional. Invent. Math. 101, 425-448 (1990) Zbl 0724.35040 MR 1062970

[25] Urbas, J. I. E.: On the existence of nonclassical solutions for two classes of fully nonlinear elliptic equations. Indiana Univ. Math. J. 39, 355-382 (1990) Zbl 0724.35028 MR 1089043

[26] J. Wang, X.: A class of fully nonlinear equations and related functionals. Indiana Univ. Math. J. 43, 25-54 (1994) Zbl 0805.35036 MR 1275451 